\title{
A Review on Techniques for Diagnosing and Monitoring Patients with Parkinson's Disease
}

Prasad RKA, Babu SS, Siddaiah N and Rao KS*

Department of ECE, KL University, Green Fields, Guntur, Andhra Pradesh, India

\begin{abstract}
Parkinson's disease (PD) is the second highest neurodegenerative disease that impairs speech besides causing bodykinesia, postural instability, rigidity and tremor. From the present research works it is understood that the disease is the result of lack of dopamine in brain cells. However, its real cause is yet to be established satisfactorily. In this paper we review the present state of the art of the PD including the nature of disease, its characteristics, the treatment, algorithms or techniques available to diagnose or monitor the patients with the disease, sensors and wearable devices being used to help PD patients and physicians. We also explored many speech based solutions to the disease. The essence of our review is that this neurodegenerative disorder needs further research and development of wearable devices that can help patients to lead a normal life. In this paper we provide the summary of findings which leads to continue our research to develop a wearable device with state of the art software that can help PD patients to have better lives.
\end{abstract}

Keywords: Parkinson's disease; Neurodegenerative disease; Wearable devices; Bodykinesia

\section{Introduction}

Parkinson's disease (PD) is a disease pertaining to central nervous system. It is a degenerative disorder that impairs speech, motor skills and other functions of the human who suffers from it [1]. The disease is the result of lack of dopamine in brain cells of human being and reflects it in the form of motor complications. The reason for this disease is not yet clearly established. The PD progress is slow and the progression may take many years. There is limited medical treatment to $\mathrm{PD}$ patients which can reduce disease symptoms [2]. There are four important symptoms of PD such as bodykinesia, postural instability, rigidity and tremor. In order to classify Parkinson's disease based on the bodykinesia there are three approaches known as foot pressure analysis, finger motion analysis and the Unified Parkinson's Disease Rating Scale (UPDRS) [3]. The PD is in the second place among the neurodegenerative affliction. The first place is occupied by Alzheimer's disease (AD). There is a life risk of $1.3 \%$ for women and $2 \%$ for men as per the study of Olmstead country. The disease is going to increase in the future to come due to the medical expenses and other reasons [4].

The Clinical Symptoms of Parkinson's disease are Tremor or shivering, Small Hand Writing, Loss of Smell, Trouble in Sleeping, Trouble moving, Constipation, Soft of Low voice, Masked Face, Dizzying or Fainting and Stooping or Hunching over as shown in Figure $1[5]$.

Much attention in the research circles was there on PD. Broadly the research was into different areas including the development of algorithms and techniques to diagnose or monitor the progression of disease, wearable devices and sensors and their role for PD diagnosis and monitoring, and the speech based solutions pertaining to PD. The algorithms or techniques were explored in reference [1-4,6,7]. The techniques or algorithms focused by these research papers include Probabilistic Neural Network (PNN) and its variants, rule based decision algorithm, weighted fuzzy membership functions, Artificial Neural Networks (ANNs) and Support Vector Machines (SVM), Evolutionary Algorithms (EL), and algorithms based on Hidden Markov Model (HMM) [1-4,6].

With respect to the wearable devices and sensors to predict or

diagnose or monitor PD in patients there were plethora of research activities explored in [8-14]. FOTIADIS et al. [14] studied the importance of wearable devices in monitoring patients in order to diagnose or help medical staff and patient's relatives to take steps required. El-Gohary et al. [8] used internal sensors for accurate assessment of symptoms of PD in patients. Chen et al. [9] built a web based application by name "Mercury Live" which is connected to wearable devices that is used to monitor patients with PD. Sant'Anna et al. [10] studied the usage of internal sensors in observing patients with PD. Lorincz et al. [11] studied the sensor networks that help in monitoring signs of patients. Manto et al. [12] studied the tremor and its characteristics. Lorincz et

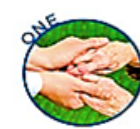

TREMOROR SHAKING

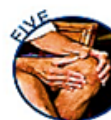

TROUBLE MOVING

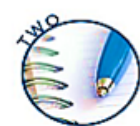

SMALL HAND WRITING

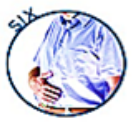

CONSTIPATION

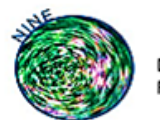
FAINTING

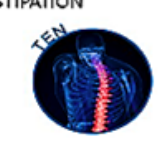

Figure 1: Clinical symptoms of PD [5]

*Corresponding author: Srinivasa Rao, Research Scholar, Department of ECE KL University, Green Fields, Guntur, Andhra Pradesh, India, Tel: 08662577715; E-mail: srinivasakarumuri@gmail.com

Received February 22, 2016; Accepted March 21, 2016; Published March 31 2016

Citation: Prasad RKA, Babu SS, Siddaiah N, Rao KS (2016) A Review on Techniques for Diagnosing and Monitoring Patients with Parkinson's Disease. J Biosens Bioelectron 7: 203. doi:10.4172/2155-6210.1000203

Copyright: (c) 2016 Prasad RKA, et al. This is an open-access article distributed under the terms of the Creative Commons Attribution License, which permits unrestricted use, distribution, and reproduction in any medium, provided the original author and source are credited. 
al. [11] describes a network made up of wireless and wearable sensors that help in motion analysis of patients with diseases like PD, stroke, neuromotor disorders and epilepsy. Darwish and Hassanien [13] studied solutions for healthcare monitoring. Especially they focused on the wearable and implantable body sensor networks (WIBSN).

Speech based solutions to PD were explored in reference [15-26]. Many of these researches divided the voice into two type's namely normal voice and pathological voice. The common thread in all these researches is that the researchers identified the relation between the changes in voice and the PD. Our contribution in this paper includes the review of various research papers pertaining to the second highest neurodegenerative disease (i.e. PD) that still needs improvements in diagnosis and medical aids. We further explore in making a device that can help PD patients to live happily in spite of the disease. The remaining paper is structured as follows. Section II reviews the PD related researches in terms of algorithms, techniques, the usage and role of sensors and wearable devices besides exploring the speech based solutions. Section III summarizes findings while section IV concludes the paper.

\section{Background Works}

This section reviews literature pertaining to Parkinson's disease in terms of algorithms and techniques used to diagnose or monitor PD, the role of sensors or wearable devices in treating PD and the speech based solutions for PD.

\section{Algorithms or techniques diagnose or monitor PD}

Ene studied the application of neural network based approaches to identify the people from those suffering from Parkinson's disease [1]. Especially Ene applied probabilistic neural network $(\mathrm{PNN})$ variants to investigate the differences between people with Parkinson's disease and healthy people. Ene applied various search methods such as Monte Carlo Search (MCS), Incremental Search (IS) and Hybrid Search (HS). From his studies it is evident that the accuracy between $79 \%$ and $81 \%$ of Parkinson's disease. Kostek et al. [2] presented a novel methodology that makes use of rule-based decision algorithm to predict the status of Parkinson's disease in patients. The methodology is named as "Unified Parkinson's Disease Rating Scale (UPDRS)". This methodology has resulted in most suitable for automatic assessment of state of PD in patients.

Tian et al. proposed a method that can classify PD patients. Weighted fuzzy membership functions are used by them with minimal features in order to classify PD patients accurately [3]. This approach resulted in higher accuracy of $92.82 \%$, specificity $85.42 \%$ and performance sensitivity $95.24 \%$. Gil and Johnson applied support vector machines (SVMs) and artificial neural networks (ANNs) for diagnosing Parkinson disease in patients. This approach resulted in 90\% accuracy [4]. The classifiers constructed based on the two kinds of techniques were evaluated and proved that they are useful in diagnosing PD. Lones et al. studied the movement characteristics of patients with PD [6]. The researchers used evolutionary algorithms in order generate classifiers that are used to know the movement characteristics of patients with PD. The patterns thus obtained are used in clinical treatment of PD patients. These classifiers achieved $95 \%$ accuracy in diagnosing PD.

Pavel et al. studied patient's movements to know the characteristics of PD [7]. They proposed a set of algorithms that are used to assess the neurological state of the people with PD. Their diagnosis model is based on Hidden Markov Model (HMM). This model was used to assess variability and gait velocity in the people with $\mathrm{PD}$.

\section{The role of wearable devices to monitor Parkinson's disease}

FOTIADIS et al. studied the importance of wearable devices in monitoring patients in order to diagnose or help medical staff and patient's relatives to take steps required [14]. These devices take patients' vital signs as input and process them in order to visualize or inform the concerned people to take remedial steps. With these devices it is possible for the patients to reduce medical expenses as they are monitored being comfort at the home.

El-Gohary et al. used internal sensors for accurate assessment of symptoms of PD in patients [8]. Especially these sensors provide data that can be used to monitor movement disorders which are crucial in diagnosing PD and provide further clinical trials. Instead using manual clinical evaluation, these researchers introduced internal sensors that can automate the monitoring of tremor fluctuations and help in determining the dosage of drugs and so on as shown in Figure 2 [8].

Chen et al. built a web based application that is used to monitor patients with PD [9]. Their application is known as "Mercury Live" which has three tiers such as a data collection engine that make use of wearable sensors to take patients' vital signs, web services for live streaming of the data that has been collected through wearable devices, and web based user interface that allows users to have interaction with application with live video conferencing capabilities. Sant'Anna et al. studied the usage of internal sensors in observing patients with PD [10]. They introduced symbol based symmetry index in the early detection of Parkinson's disease. The sensors computed such symmetric index that helped in monitoring disease and diagnose in early stages. Thus the researchers produced a new measure of movement symmetry that helped in early detection of PD in patients. Their application has shown high specificity and sensitivity.

Lo et al. studied the sensor networks which help in monitoring signs of patients [11]. They presented a scheme that helps in localization of sensor nodes which gives an understanding about the validation of localization scheme. The network they built is used to monitor the performance of wearable sensor devices that are associated with human body. Moreover their network can monitor the patient's falllike motions and discriminates them from actual falls. This helps in diagnosing diseases like PD.

Manto et al. studied the tremor and its characteristics [12]. Besides, they investigated the wearable devices that helped in suppression of upper-limb tremor while patient has natural movement. The researchers succeeded in establishing constraints and parameters for making a truly working active orthoses. To achieve this they have applied the knowledge of signal processing and control technologies, biomechanics, neural control of movement and neurological sciences.

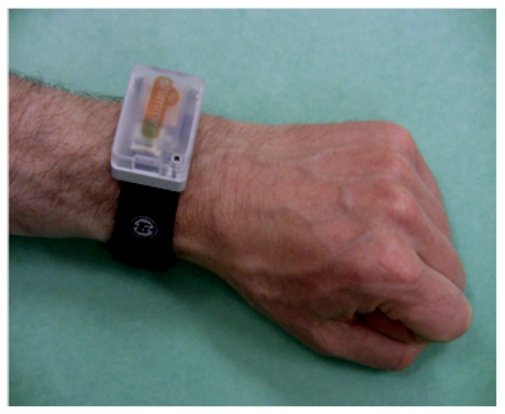

Figure 2: Model of inertial sensor used [8]. 
Citation: Prasad RKA, Babu SS, Siddaiah N, Rao KS (2016) A Review on Techniques for Diagnosing and Monitoring Patients with Parkinson's Disease. J Biosens Bioelectron 7: 203. doi:10.4172/2155-6210.1000203

Lorincz et al. describes a network made up of wireless and wearable sensors which help in motion analysis of patients with diseases like PD, stroke, neuromotor disorders and epilepsy [13]. The sensor network platform built by researchers is known as "Mercury". This network is made up of eight wearable devices that are associated with patient's body. These devices have long batter life and capable of monitoring 12 to 18 hours continuously. The platform combines wireless and wearable sensors to work together in diagnosing and monitoring patients with PD and other such diseases.

Darwish and Hassanien studied solutions for healthcare monitoring [13]. Especially they focused on the wearable and implantable body sensor networks (WIBSN) that are used to diagnose and monitor diseases like PD as shown in Figure 3. They further advocated the usage of telecommunication technologies such as Wi-MAX, Wi-Fi and $3 \mathrm{G}$ to work in tandem with telemedicine equipment. Figure 2 shows the importance of inter-disciplinary sciences for the success of WIBSNs in future [8].

As can be seen in Figure 3 [13], it is evident that the future of WIBSNs needs the help of inter-disciplinary sciences that help in diagnosing or monitoring patients with PD or other diseases.

Mario PANSRA et al. made a system for continues assessment and monitoring of motor status in Parkinson's disease by comparing entropy based gait [26]. They experimented with 8 patients in whom four are PD patients ( 3 male and 1 female); four are healthy people ( 3 male and 1 female).

They placed 4 accelerometers, two are at left and right forearms, two are at left and right calves and the fifth is at trunk. The results were collected and processed using SHIMMER platform as shown in Figure $4[26]$.

Ron seng chang et al. experimented with Laser lines and CMOS (complementary Metal Oxide) Sensors to detect the dorsum manus

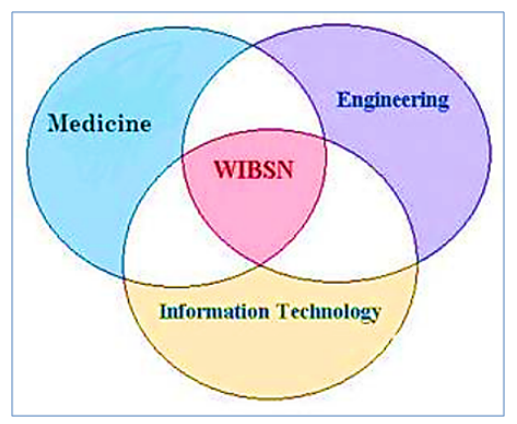

Figure 3: Interdisciplinary sciences required by WIBSNs in future [13]

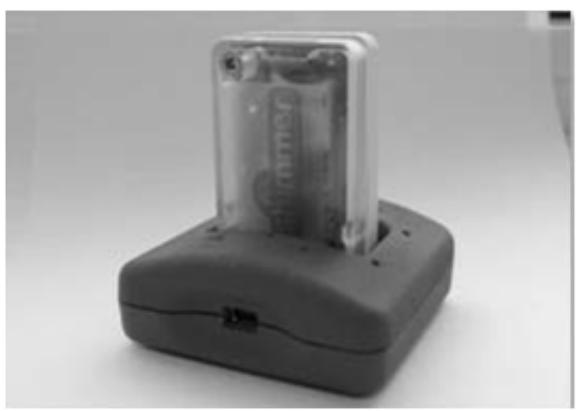

Figure 4: Shimmer Device [26] vibrations in rest and postural situations [27]. The arrangement and the 3D and 2D Structures are shown in Figures 5 and 6 [27].

The experiment is carried out for the duration of 20 seconds. The Laser light is able to fall on the dorsum manus and the complementary metal oxide semiconductor image sensor continuously records the LASER line as shown in Figure 7 [27]. By Triangulation and centroid calculation on the surface of the dorsum manus vibration, they have detected the involuntary trembling of the dorsum manus in the time domain. The accuracy of the work is about $95 \%$ suited to determine the stage Parkinson's disease [27,28].

Bustamante Bello et al. prepared a low cost tool for characterization and detection of some neurodegenerative diseases by the movement of tremor of muscle in patient finger which reduces the number of clinical tests [29]. The device is like a glove as shown in Figure 8 which consists of 4 accelerometers which are connected to fingers, and one attached to wrist [29]. The setup for the accelerometer is shown in Figure 9 [29]. The time taken to conduct the tests is 20 seconds only and the accuracy of the system is about $95 \%$. The Output result was shown in Figure 10 [29].
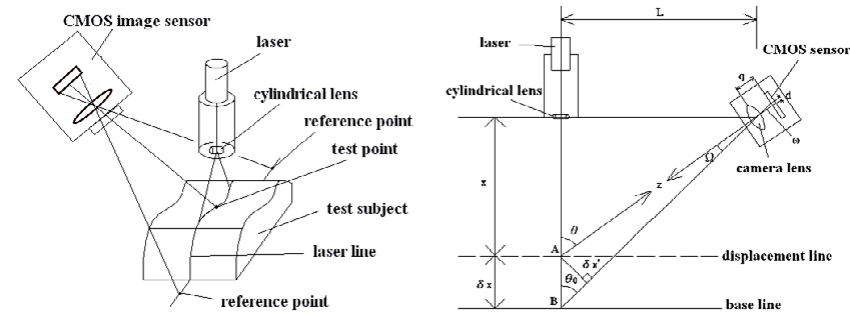

Figure 5: Geometrical layout of the Parkinson's disease patient arm vibration measurement system 3D layout, 2D layout [27].

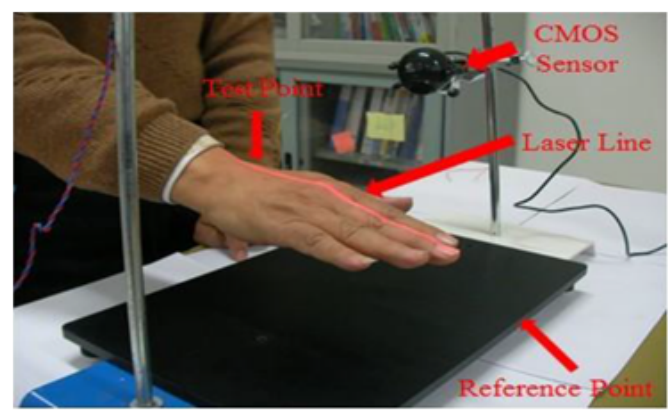

Figure 6: Hand detection method for postural action tremors [27].

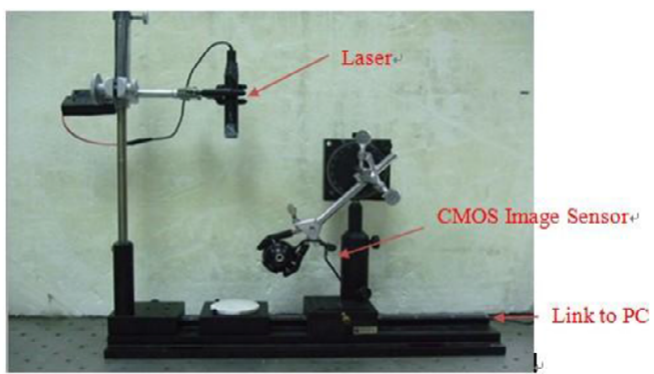

Figure 7: Actual implementation of the proposed system [27] 


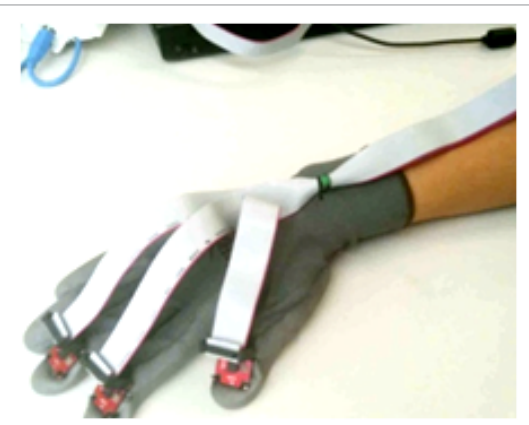

Figure 8: Glove with accelerometers attached to fingertips [29].

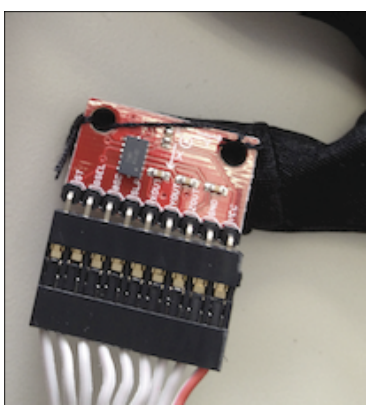

Figure 9: MMA7361 Accelerometer attached to glove's fingertip [29].
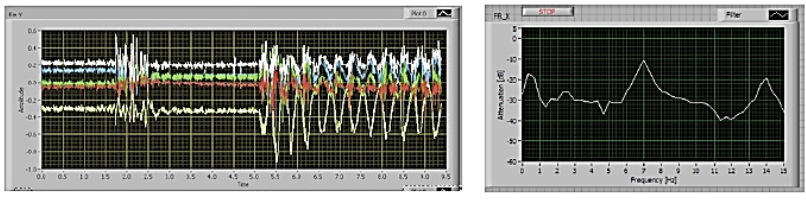

(a) Time and (b) frequency spectrum of the average of the $x$-axis signal

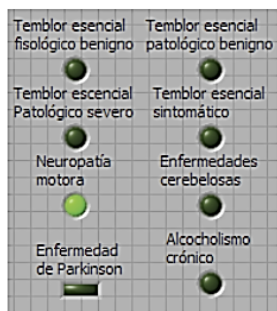

Figure 10: Output signal using platform [29]

ERocon et al. proposed the robot based wearable sensor in finding tremor of human hand (WOTAS) as shown in Figure 11 [30]. The Robotics involved in this technique are to be modified further and actuators are the one that probably most limit wear ability and probability at the present time while sensors are the most limiting efficient implementation of cHRI and pHRI [30].

Barth et al. designed a sensor-based system to measure the movement of the upper and lower extremities would therefore complement the clinical evaluation of PD [31]. In this study two different sensor-based systems were combined to assess movement of $18 \mathrm{PD}$ patients and 17 healthy controls. First, hand motor function was evaluated using a sensor pen with integrated accelerometers and pressure sensors as shown in Figure 12A [31], and second, gait function was assessed using a sports shoe with attached inertial sensors (gyroscopes, accelerometers) as shown in Figure 12B [31]. Subjects performed standardized tests for both extremities. Features were calculated from sensor signals to differentiate between patients and controls.

Ming Fang Chang et al. have designed a walker (CAIROW) shown in Figure 13 [28] to identify the Parkinson`s Disease and which helps to walk carefully. They have experimented with 9 elder with PD for 6 days and observed the physical changes. The patients used to walk for 20 meter long with the vehicle. The People with Parkinson's disease are not able to walk properly

Elsayed et al. has experimented with movement related tests and movement gait analysis to identify the persons suffering with Parkinson's disease and they place some ear worn cameras to observe the patient condition continuously [32].

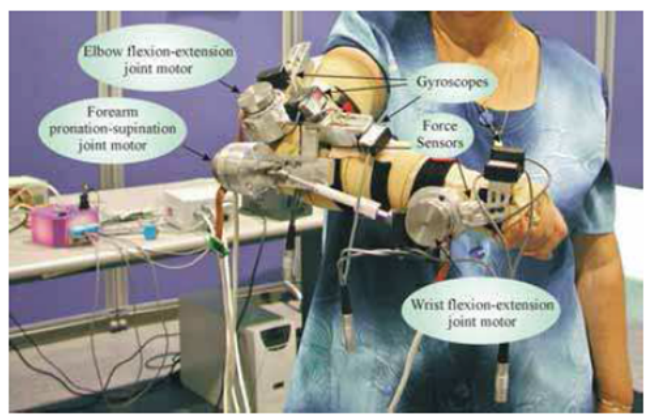

Figure 11: Patient using WOTAS at different parts of the hand [30].

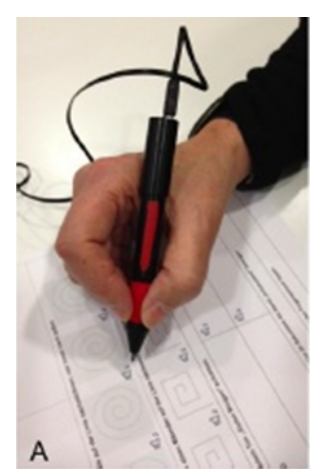

Figure 12a: Biometric Smart Pen (BiSP) for analysis of hand motor function [31].

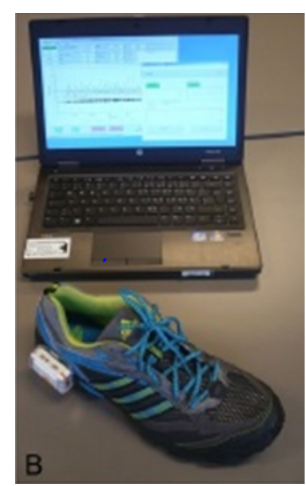

Figure 12b: Sensor Shoe Setup [31]. 


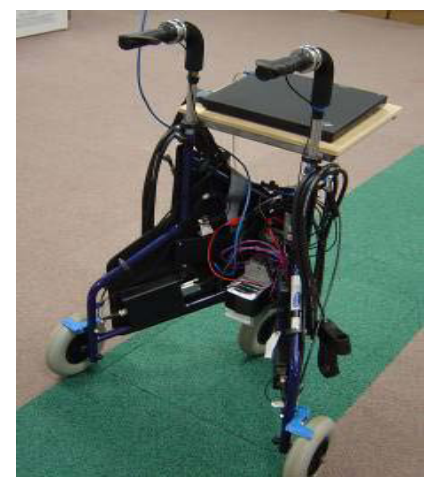

Figure 13: Context aware assisted interactive Robotic Walker [28]

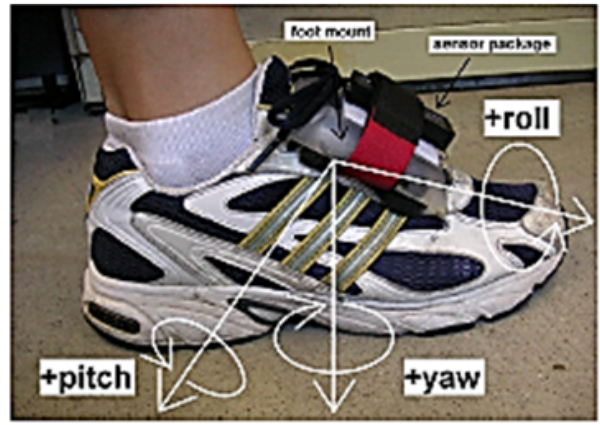

Figure 14: Orientation of axes on foot with sensor package attached using foot mount and sample 3D displacement signal in pitch, roll, and yaw directions over a 10-second segment [35]

Shyamal patel et al. have implemented a system known as SVM (Support Vector Machines) to predict the clinical features of the severity of Parkinson's Disease [33]. From the Sensor Data they have predicted the optimal window length [34]. The accuracy of the Work is about $75 \%$.

Ahamed AI jawad et al. predicted the Parkinson's disease by TUG (The Time Up and Go) [34]. In this method they proposed some activities like Sit to Stand, 3-meters walk, 180 degrees turn, walk back, another turn and sit on chair. While they perform the above activities they record the movements and predict the severity of the Parkinson's disease.

Iris Tien et al. used wireless sensor i to identify the gait abnormalities in Parkinson's disease. They obtained the physical features of Pitch, roll and yaw rotations of the foot during walking and they have used the component analysis to select the features and they classified the sensor data [5]. The Example of placement of sensor and data that was processed during the course of experiment is shown n Figure 14 [35].

Mark et al. [35], estimated the posture from movements without requiring integrating over time and the resulting complications as shown in Figure 15 [35]. Tremor of the hand is affected by posture in an intuitive way and therefore can be used to estimate the posture of the arm. They recorded the posture and tremor of the arms of the volunteers. They used the mirror axis in the covariance of the hand tremor. They estimated the angle of the forearm with a standard deviation of $4^{\circ}$ and for the elbow at rest is $10^{\circ}$.

\section{Speech Based Observations to Parkinson's Disease}

Tsanas et al. studied on speech of patients with PD [15]. They conducted speech tests to patients in order to measure the progression of PD in them. They could able to monitor patients suffering from PD with 95\% accuracy. Their work helps the medical staff to treat PD patients appropriately as the system is capable of accurately finding the progression of the disease in them. Much speech related measures are used by these researchers in order to provide accurate results of the observations made on PD patients through speech.

Khan also studied the speech anomalies to recognize the PD in patients [16]. Rosen et al. identified the relationship in phonetic variation and PD in patients [17]. McRae and Tjaden studied speech anomalies and characterized the relationship between the perceptual impressions of PD patients and their acoustic output [18]. Especially they studied on the vowels such as 'i', 'u', 'a', 'f', and's'. There are two speaker groups in PD patients known as HC and HKD. According to Kleinow and Smith the loud speech and habitual speech of patients are closer [19]. Similar conclusions are made by Neel [20] and Tjaden [21]. These researches are boiled down to categorize between normal voice and pathological voice. Wavelet analysis on speech of PD patients made to visualizes the difference between both kinds of voices as shown in Figure 16 [16,22].

From Figure 16, it is evident that the voice of the PD patients can help in classification of them using tools like ANN. In the speech of PD patients were analyzed and classified into F1 and F2 based on the frequencies. These two are mutually opposite as F1 increases, F2 decreases. However they are same when libs are rounded. Continuous sound analysis of PD patient is made by Izworski et al. for recognizing HKD kind of voice automatically [23].

ANN was used as statistical tool by Mehmet et al. to recognize the speech abnormalities based on the voice features of PD patients [24]. Especially a fuzzy logic based ANN was used to find the difference between the normal voice and pathological voice. This approach
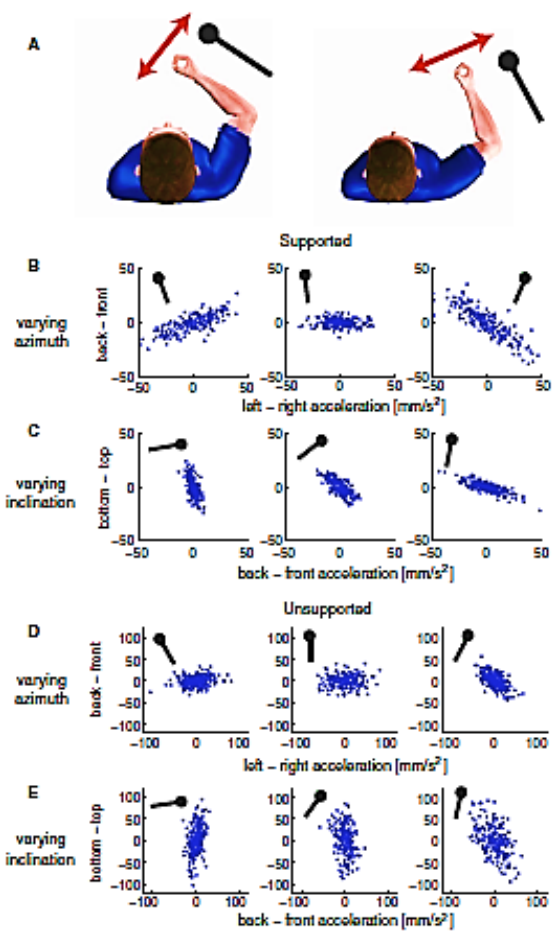

Figure 15: Posture positions of the arms [35]. 
Citation: Prasad RKA, Babu SS, Siddaiah N, Rao KS (2016) A Review on Techniques for Diagnosing and Monitoring Patients with Parkinson's Disease. J Biosens Bioelectron 7: 203. doi:10.4172/2155-6210.1000203

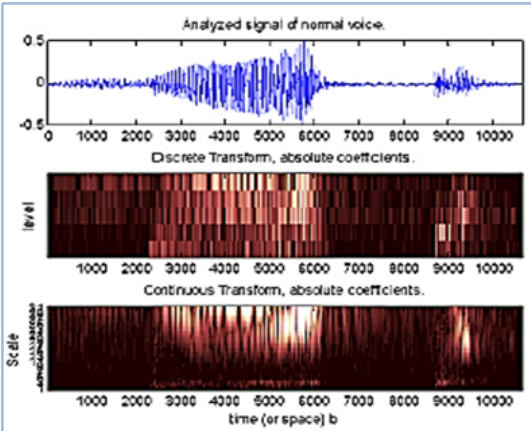

a. Normal Voice

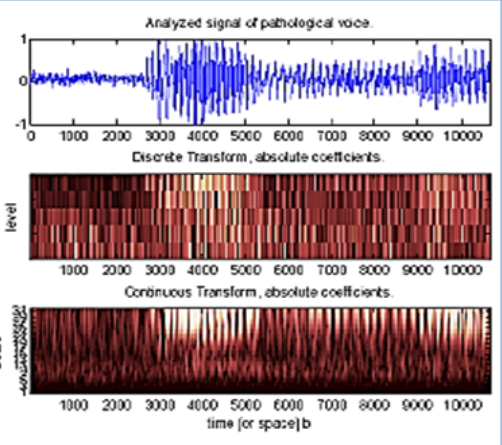

b. Pathological Voice

Figure 16: Difference between normal voice and pathological voice [16].

\begin{tabular}{|c|c|c|}
\hline Name of the Author & Techniques to monitor PD & Results \\
\hline Ene [1] & $\begin{array}{l}\text { Probabilistic Neural Network (PNN), and search methods such } \\
\text { as Monte Carlo Search (MCS), Incremental Search (IS) and } \\
\text { Hybrid Search (HS) }\end{array}$ & Accuracy between $79 \%$ and $81 \%$ \\
\hline Kostek et al. [2] & Rule based decision algorithm using the methodology UPRDS & Rough set based decision algorithm is most suitable \\
\hline Tian et al. [3] & Weighted fuzzy membership functions & $\begin{array}{l}\text { higher accuracy of } 92.82 \% \text {, specificity } 85.42 \% \text { and } \\
\text { performance sensitivity } 95.24 \%\end{array}$ \\
\hline Gil and Johnson [4] & $\begin{array}{l}\text { Support vector machines (SVMs) and artificial neural networks } \\
\text { (ANNs) }\end{array}$ & $90 \%$ accuracy \\
\hline Iris Tien et al. [5] & Wireless sensor based & $\begin{array}{l}\text { They placed the wireless sensor based sensors at } \\
\text { different places of the shoe and noted the movement } \\
\text { disorders }\end{array}$ \\
\hline Lones et al. [6] & Evolutionary algorithms & $95 \%$ accuracy \\
\hline Pavel et al. [7] & Algorithms based on Hidden Markov Model (HMM) & Ability to assess gait velocity and its Variability \\
\hline Fotiadis et al. [14] & Wearable devices & Visualize the vital signs of patients \\
\hline El-Gohary et al. [8] & Internal sensors & Ability to track tremor in PD patients \\
\hline Chen et al. [9] & $\begin{array}{l}\text { Mercury Live, a web based application working in tandem with } \\
\text { wearable devices }\end{array}$ & Able to monitor PD \\
\hline Sant'Anna et al. [10] & $\begin{array}{l}\text { Introduced symbol based symmetry index in the early } \\
\text { detection of Parkinson's disease }\end{array}$ & High specificity and sensitivity \\
\hline Lorincz et al. [11] & Sensor network platform & $\begin{array}{l}\text { Monitor the patients' fall-like motions and discriminates } \\
\text { them from actual falls }\end{array}$ \\
\hline Lorincz et al. [11] & Wearable devices & Motion analysis of patients \\
\hline Manto et al. [12] & Wearable devices & Helped in suppression of upper-limb tremor \\
\hline Darwish and Hassanien [13] & Wearable and implantable body sensor networks (WIBSN) & More accuracy is achieved with inter-disciplinary sciences \\
\hline Mario PANSRA et al. [26] & SHIMMER PLOTFORM & $\begin{array}{l}\text { Results were collected on shimmer platform and } \\
\text { Processed the accuracy they got is about } 85 \%\end{array}$ \\
\hline Ron seng chang et al. [27] & Laser Lines and CMOS Sensor & $\begin{array}{l}\text { Using LASER lines they calculated the resting tremor and } \\
\text { the sensor is placed in shoe to identify the movement } \\
\text { disorder The accuracy they got is about } 90 \%\end{array}$ \\
\hline Bustamante bello et al. [29] & Gloves with Accelerometers & $\begin{array}{l}\text { Accelerometers Embedded in Gloves to identify the } \\
\text { resting tremor }\end{array}$ \\
\hline ERocon et al. [30] & Robot based Wearable sensor(WOTAS) & $\begin{array}{l}\text { They fixed the accelerometer sensors at different places } \\
\text { of the hand and observed the tremor of the hand. }\end{array}$ \\
\hline Barth j et al.[31] & Sensor based Pen & $\begin{array}{l}\text { By the variation in the hand writing, which was observed } \\
\text { by sensor based pen }\end{array}$ \\
\hline Ming Fing Chang [28] & CAIROW (Context aware assisted interactive Robotic Walker) & $\begin{array}{l}\text { The severity of Parkinson's disease can be identified by } \\
\text { walk test }\end{array}$ \\
\hline M. Elsayed [32] & Ubiquitous Health care Monitoring & By the impairment of walking gait \\
\hline Shamal patel et al. & Support vector Machne & $\begin{array}{l}\text { By the sensor data they calculated the window length and } \\
\text { checked the severity of the Parkinson's Disease. }\end{array}$ \\
\hline Ahmed Al jawed [33] & TUG - TIME UP AND GO & Walk test to predict parkinson's Disease \\
\hline Mark V et al. [35] & Determining posture from physiological tremor & The accuracy is about $75-80 \%$ \\
\hline References [15-25] & Speech based solutions & $\begin{array}{l}\text { Found relation between PD and the change in phonetics } \\
\text { of patients }\end{array}$ \\
\hline
\end{tabular}

Table 1: Summary of research findings. 
Citation: Prasad RKA, Babu SS, Siddaiah N, Rao KS (2016) A Review on Techniques for Diagnosing and Monitoring Patients with Parkinson's Disease. J Biosens Bioelectron 7: 203. doi:10.4172/2155-6210.1000203

Page 7 of 7

has achieved $94.35 \%$ accuracy. Salhi et al. studied the normal and pathological voices of PD patients using linear predictive coding approach in order to monitor the progression of PD in patients [24].

\section{Summary of Findings}

This section summarizes the findings pertaining to Parkinson's disease. The summary includes the algorithms, techniques, wearable devices and sensor usage in combating the diseases besides the speech based solutions that existed for diagnosing and monitoring PD.

As seen in Table 1, it is evident that much work is done by many researchers on algorithms, techniques, sensor networks, wearable devices, besides speech based solutions for Parkinson's disease.

\section{Conclusion}

In the above literature survey, we observed the facts on Parkinson's disease. Our focus was on the disease, its causes, symptoms and the solutions available. An important observation is that the causes of the disease are not yet satisfactorily established. The accuracy to identify and to measure the condition of the disease, the above said techniques gives only $80-85 \%$ on an average. To increase the accuracy and to identify at an early stage our research is progressing. Towards this end we continue our research in future to invent a wearable device with state of the art software which can help PD patients to lead normal life. In this paper, we explored various algorithms, techniques, sensors, wearable devices, and speech based solutions to Parkinson's disease in terms of disease diagnosis and monitoring of patients with PD. We also summarized the findings on the research work done by several researchers on Parkinson's disease.

\section{References}

1. Ene M (2008) Neural network-based approach to discriminate healthy people from those with Parkinson's disease. 35: 112-116.

2. Kostek B, Kaszuba K, Zwan P, Robowski P, Slawek J (2012) Automatic assessment of the motor state of the Parkinson's disease patient-a case study. Diagnostic Pathology 7: 1-8.

3. Tian XW, Lim JS, Zhang ZX, Kim Y, Lee H, and Lee SH (2011) Minimum Feature Selection for Telemonitoring of Parkinson's Disease. ICCSIT pp: 33-36.

4. David Gil A, Johnson MB (2004) Diagnosing Parkinson by using Artificial Neural Networks and Support Vector Machines. Global Journal of Computer Science and Technology pp: 63-9.

5. Tien I, Glaser SD, Aminoff MJ (2010) Characterization of Gait Abnormalities in Parkinson's Disease Usinga Wireless Inertial Sensor System. 32nd Annual International Conference of the IEEE EMBS pp: 3353-3356.

6. Lones MA, Smith SL, Alty JE, Lacy SE, Possin KL, et al. (2012) Evolving Classifiers to Recognise the Movement Characteristics of Parkinson's Disease Patients. IEEE pp: 559-576.

7. Pavel M, Hayes T, Tsay I, Erdogmu D, Paul A, et al. (2006) Continuous Assessment of Gait Velocity in Parkinson's Disease from Unobtrusive Measurements pp: 1-4.

8. El-Gohary M, McNames J, Chung K, Aboy M, Salarian A, et al. (2010) Continuous At-Home Monitoring of Tremor in Patients with Parkinson's Disease pp: 420-424.

9. Chen B, Patel S, Buckley T, Rednic R, McClure DJ, et al. (2011) A Web-Based System for Home Monitoring of Patients With Parkinson's Disease Using Wearable Sensors 58: 831-836

10. Sant'Anna A, Salarian A (2011) A New Measure of Movement Symmetry in Early Parkinson's Disease Patients Using Symbolic Processing of Inertial Sensor Data 58: 2127-2135.

11. Lorincz K, Chen B, Challen GW, Chowdhury AR, Patel S, et al. (2009) Mercury: A Wearable Sensor Network Platform for High-Fidelity Motion Analysis pp: 1-14

12. Manto M, Topping M, Soede M, Sanchez-Lacuesta J, Harwin W, et al. (2003)
Dynamically Responsive Intervention for Tremor 22: 120-132

13. Darwish A, Hassanien AE (2011) Wearable and Implantable Wireless Sensor Network Solutions for Healthcare Monitoring. Sensors 11: 5561-5595.

14. Fotiadis DI, Glaros C, Likas A (2001) Wearable Medical Devices.

15. Little MA, McSharry PE, Hunter EJ, Spielman J, Ramig LO (2003) Suitability of dysphonia measurements for telemonitoring of Parkinson's diseases 56: 1015-1022.

16. Khan T (2008) Parkinson's Disease Assessment Using Speech Anomalies: A Review pp: 1-6.

17. Rosen KM, Kent RD, Delaney AL, Duffy JR (2006) Parametric Quantitative Acoustic Analysis of Conversation Produced by Speakers with Dysarthria and Healthy Speakers. J Speech Lang Hear Res 49: 395-411.

18. Salhi L, Talbi M, Cherif A (2008) Voice Disorders Identification Using Hybrid Approach: Wavelet Analysis and Multilayer Neural Networks. WASET 2: 3005-3012.

19. Kleinow J, Smith A, Ramig LO (2001) Speech Motor Stability in IPD: Effects of Rate and Loudness Manipulations. J Speech Lang Hear Res 44: 1041- 1051.

20. Neel AT (2009) Effects of Loud and Amplified Speech on Sentence and Word Intelligibility in Parkinson Disease. J Speech Lang Hear Res 52: 1021-1033.

21. Tjaden K, Wilding GE (2004) Rate and Loudness Manipulations in Dysarthria: Acoustic and Perceptual Findings. J Speech Lang Hear Res 47: 766-783.

22. Letter DM, Santens P, Bodt DM, Boon P, Borsel JV (2006) Levodopa induced alterations in speech rate in advanced Parkinson's disease. Acta Neurol Belg 106: 19-22.

23. Izworski A, Augustyniak P, Orzechowski T (2006) Processing and analysis of voice anomalies in course of Parkinson's diseases. In Proceedings of Eighth IASTED (Honolulu, Hawaii, USA, 14-16 August) pp: 354-357.

24. Caglar MF, Cetisli B, Toprak IB (2010) Automatic Recognition of Parkinson's disease from Sustained Phonation Tests Using ANN and Adaptive NeuroFuzzy Classifier. J Speech Lang Hear Res 1: 59-64.

25. Fahn S (1987) UPDRS. Unified Parkinson Disease Rating Scale. In: Fahn S, Marsden CD, Calne DB, Goldstein M, (eds.) Recent developments in Parkinson's disease. Florham Park, Macmillan Healthcare Information, New Jersey pp: 153-163.

26. Pansera M, Estrada JJ, Pastor L, Cancela J Greenlaw R, et al. (2009) Mult parametric system for the continues assessment and monitoring of motor status in Parkinson's disease: an entropy based gait comparison IEEE EBMS pp: $1242-1245$

27. Chang RS, Chiu JH, Chen FP, Chen JC, Yang JL (2011) A Parkinson's Disease Measurement System Using Laser Lines and a CMOS Image Sensor Sensors 11: $1461-1475$.

28. Chang MF (2012) Design and implementation of an active robotic walker for Parkinson's Patients SICE Annual Conference pp: 2068-2073.

29. Bello RB Diagnostic Aid System based on triaxial high sensitivity MEMS for detection and characterization of specific neurodegenerative diseases. Workshop Proceedings of the 8th International Conference on Intelligent Environments pp: $2-47$

30. Rocon E, Moreno JC, Gallego JA, Pons JL (2009) Wearable Robots in Rehabilation Engineering Tremor Suspension. Rehabilation Engineering pp: 203-223.

31. Barth J, Rodriguez AER, Perez LY (2012) Combined analysis of sensor data from hand and gait motor function improves automatic recognition of Parkinson's disease. IEEE-EMBS pp: 5122-5125.

32. Sayed ELM, Alsebai A, Salaheldin A, Gayar NE, Body and Visual Senso Fusion for motion Analysis in Ubiquitous Health care systems. IEEE internation conference pp: $250-254$

33. Patel S, Hughes R, Huggins N, Standaert D (2008) Using wearable sensors to predict the severity of symptoms and motor complications in Late Stage Parkinson's Disease. 30th Annual International Conference of the IEEE, Engineering in Medicine and Biology Society, Vancouver pp: 3686-3689.

34. Jawad AAI, Adame MR, Romanoves M, Hobert M et.al. (2012) Using multidimensional dynamic time warping for TUG test instrumentation with inertial sensors. IEEE conference pp: 212-218.

35. Mark V, Kording KP (2011) Determining posture from physiological tremor 215 247-255. 